\title{
Sentinel Lymph Node Detection in Patients with Oral Cancer by MR Lymphography using Superparamagnetic Iron Oxide
}

\author{
Hirokazu Uemura ${ }^{1,3}$, Ichiro Ota ${ }^{*}, 2$, Takashi Fujii ${ }^{3}$, Motoyuki Suzuki ${ }^{3}$, Mio Sakai ${ }^{4}$, Katsuyuki Nakanishi ${ }^{4}$, \\ Yasuhiko Tomita ${ }^{5}$, Atsushi Noguchi ${ }^{6}$, Hiroshi Hosoi ${ }^{2}$ and Kunitoshi Yoshino ${ }^{3}$
} ${ }^{1}$ Department of Otorhinolaryngology, Saiseikai Chuwa Hospital, Japan; ${ }^{2}$ Department of Otolaryngology-Head and
Neck Surgery, Nara Medical University, Japan; Departments of ${ }^{3}$ Head and Neck Surgery, ${ }^{4}$ Radiology, ${ }^{5}$ Pathology and
${ }^{6}$ Nuclear Medicine, Osaka Medical Center for Cancer and Cardiovascular Diseases, Japan

\begin{abstract}
The purpose of this study was to examine the feasibility of interstitial MR lymphography using Superparamagnetic Iron Oxide (SPIO) to detect sentinel lymph nodes (SLNs) in head and neck cancer. For two patients with cT2N0 squamous cell carcinoma of the tongue, the submucosal injection of SPIO as well as sentinel lymph node navigation surgery (SLNNS) was performed before undergoing surgical treatment. SPIO was used for MR lymphography and Tc-99m phytate for SPECT. We compared the images by both modalities. Berlin blue stain was also performed postoperatively to verify if SLNs had the uptake of SPIO. The lymph nodes with MR signal attenuation and those with hot spots were probed to be anatomically identical. All the lymph nodes detected as a SLN by a gamma-probe contained the blue granules from SPIO. These data suggest that SPIO can be a novel tracer for performing SLNNS and SLN biopsy (SLNB) in oral cancer patients with cN0 neck.
\end{abstract}

Keywords: Head and neck cancer, sentinel lymph node, superparamagnetic iron oxide, MR lymphography.

\section{INTRODUCTION}

The sensitivity for detecting lymphatic metastasis in patients with head and neck cancer as well as melanoma and breast carcinoma has significantly increased by the use of sentinel lymph node biopsy (SLNB) [1-4]. However, SLNB in head and neck cancer is not widely accepted in Japan so far. Recently, a retrospective study on sentinel lymph node for oral and laryngopharyngeal cancer in Japan was reported [5]. Indocyanine Green (ICG) is widely used for detecting sentinel lymph nodes (SLNs) in patients with head and neck cancer or breast cancer. In addition, lymphoscintigraphy is also used for detecting SLNs in the head and neck region.

ICG is such a small weight molecule that it can reach sentinel lymph nodes rapidly after injection around the primary tumors. Constant experience on the procedure of SLNB is necessary in order to identify and pick up the SLNs accurately. It should be difficult to search the whole neck extensively for the SLNs with ICG, while SPECT helps us identify the locations of them. Recently, the new imaging technique, such as near-infrared fluorescence imaging, may compensate the disadvantage with ICG [6-8], but it is still difficult to survey the whole neck for SLNs. Therefore, SPECT is often used at the same time with ICG for detecting SLNs.

SPIO, a negative contrast enhancing agent, can be one of the alternatives of SLNs identification. A magnetometer for

*Address correspondence to this author at the Department of OtolaryngologyHead and Neck Surgery, Nara Medical University, 840 Shijo-cho, Kashihara, Nara 634-8522, Japan; Tel: +81-744-29-8887; Fax: +81-744-246844; E-mail: iota@naramed-u.ac.jp
SPIO in SLNs is available nowadays $[9,10]$, but the comparison between distribution of SPIO by its submucosal injection and that of radioisotope labeled agents remains unclear. In the current study we examined the feasibility of interstitial MR lymphography using SPIO to detect SLNs in 2 patients with oral cancer.

\section{MATERIALS AND METHODS}

\section{Patients}

This study was approved by the Institutional Review Board of Osaka Medical Center for Cancer and Cardiovascular Diseases. Two patients with cT2N0 squamous cell carcinoma of the tongue were enrolled as the subjects of this study. Written informed consent for this study was obtained from them. They consented to the submucosal injection of SPIO as well as to sentinel lymph node navigation surgery (SLNNS) at Osaka Medical Center for Cancer and Cardiovascular Diseases. Tc-99m phytate was used as a tracer in SLNNS.

\section{Superparamagnetic Iron Oxide (SPIO)}

Risovist $^{\circledR}$ was submucosally injected around the primary tumors. It was approved in Japan in 2001 as a SPIO formulation that targeted the liver reticuloendotherial system. SPIO particles are phagocytosed by reticuloendothelial cells in the liver, resulting in negative enhancement of the liver parenchyma on T2- or T2*weighted imaging. The iron oxide core is about $5 \mathrm{~nm}$ in diameter. It is coated with carboxydextran and clustered. The size of particles is $57 \mathrm{~nm}$ respectively. The SPIO particles bind with opsonins in the vessels and about $80 \%$ of dosage is 
phagocytosed by Kupfer cells in the liver reticuloendotherial system. The SPIO particles accumulate in the lysosome granules and form cluster in the Kupffer cell, the large clusters disturb local magnetic field, shortens T2 * and decrease the signal strength of the liver. Moreover, small clusters assume the approach of the water molecule to magnetic center to be easy, and shortens T1 and T2. The decreased or increased signal strength of the liver results in improvement of neoplasm-liver contrast and it contributes to the diagnosis of the liver tumors.

The SPIO particles are phagocytosed by macrophages in lymph nodes. Therefore, the decrease of signal strength also occurs in the lymph nodes with uptake of SPIO.

\section{Injection of SPIO}

Undiluted solution of Risovist ${ }^{\circledR}$ was submucosally injected with the dose of $0.45 \mathrm{mg} / \mathrm{kg} \quad(=8 \mu \mathrm{mol} \mathrm{Fe} / \mathrm{kg},<$ $1.4 \mathrm{ml} /$ body). The mucosa around tumor was not pretreated with any local anesthetics but patients complained of no severe pain. Risovist ${ }^{\circledR}$ was injected in surroundings of the primary lesion one fourth of the volume of SPIO each.

\section{SPIO Contrast Enhanced MRI}

MRI was performed twice before and 30 minute after the submucosal injection of SPIO. MRI images were evaluated for the lymph nodes with attenuation of signal strength. Both MRI images of the pre- and post-injection were compared with each other if the lymph nodes detected by MRI anatomically coincided to SLNs detected by SPECT.

\section{Detection and Removal of SLNs During Surgical Treatment}

The flowchart about imaging studies and surgical treatment for the patients was shown in Fig. (1). We intraoperatively used the gamma probe (Neo2000 Gamma Detection System, Mammotome, Cincinnati, OH) to locate the SLNs with Tc-99m phytate. The lymph nodes with the counts of ten times as much as those of the back ground values were defined as the SLNs. These lymph nodes were immediately evaluated to verify if there was an evidence of microscopical metastasis by the frozen section when removed. The iron staining with berlin blue was done to the permanent preparation to confirm the existence of iron in the SLNs.

\section{RESULTS}

The lymph nodes that accumulated SPIO located in the same anatomical positions where the hot spots with accumulation of Tc-99m phytate were identified by SPECT in both Case 1 and Case 2. These data imply that both of them turned out to be anatomically identical (Figs. 2, 3).

To detect SLNs with SPIO, but the removed SLNs were treated with berlin blue stain, instead of the using a magnetometer intraoperatively $[9,10]$. The blue granules shown in Fig. (4) indicated the uptake of SPIO in the sinus of SLNs. All the lymph nodes detected as a SLN by a gamma-probe contained those blues granules.

\section{DISCUSSION}

Organ preservation is an important goal in treatment for head and neck cancer, and the modalities such as chemoradiation aiming at coexisting of that purpose and the therapeutic effect are widely adopted. Minimum surgical stress is expected because excellent prognosis is obtained by surgical treatment for early cancer of tongue even though the potential of cervical lymph node metastasis in $\mathrm{cN0}$ neck is focused on in T1/T2 tumors. To overcome this task, the clinical researches on SLNs in the oral cavity cancer are ongoing $[5,7,11]$.

ICG is widely used to identify SLNs intraoperatively. ICG can run rapidly through the lymphatic drainage system because its particle is small. Therefore, we need to become more experienced on SLNNS or SLNB with ICG. In addition, it is low-priced and the procedure is clear. This is why ICG is still used for SLNNS or SLNB for head and neck cancer. Recently, Photodynamic Eye (PDE) and ICG is an effective combination in order to visualize tissue perfusion and SLNs [8]. We can also use a transparent hemispherical device that is effective in order to identify the lymph nodes in the deep part of the neck by shortening the distance from the skin to the target [8].

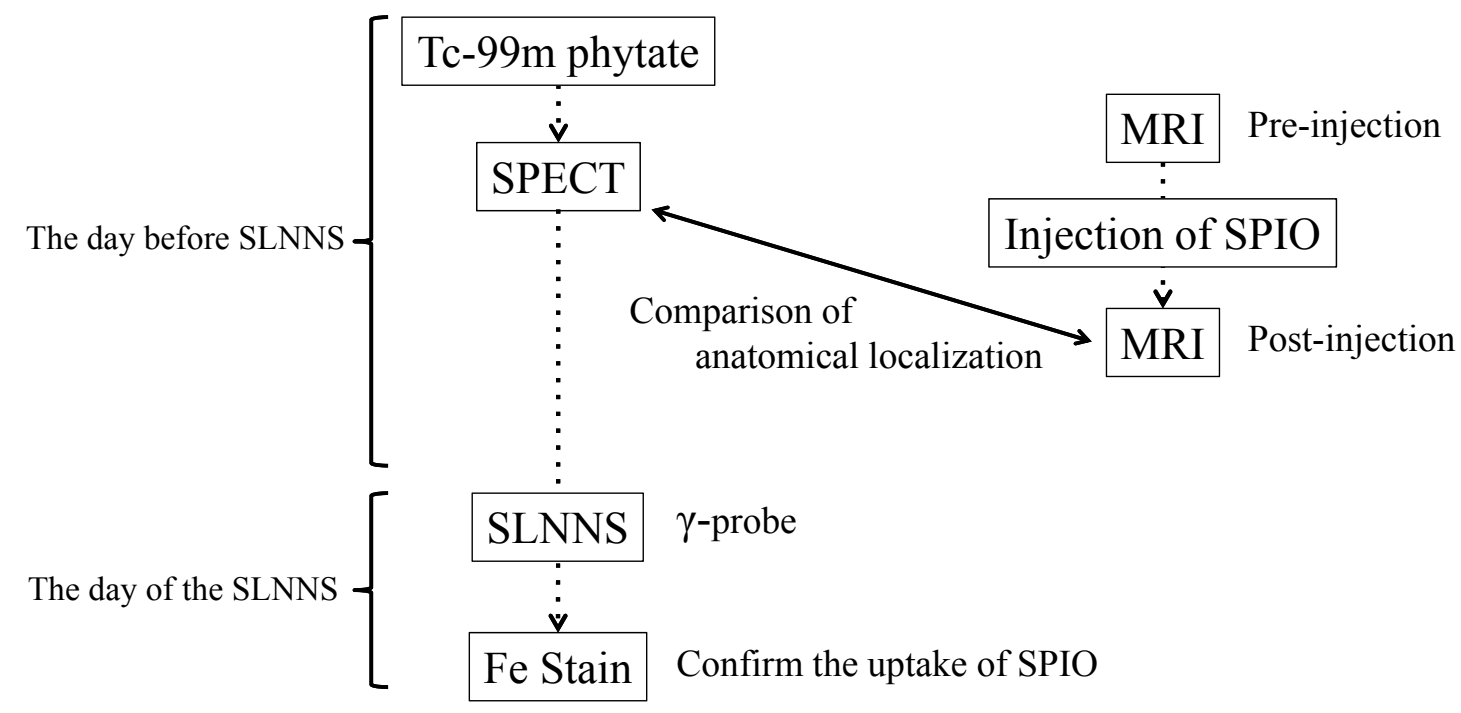

Fig. (1). The flowchart for imaging studies and SLNNS. 

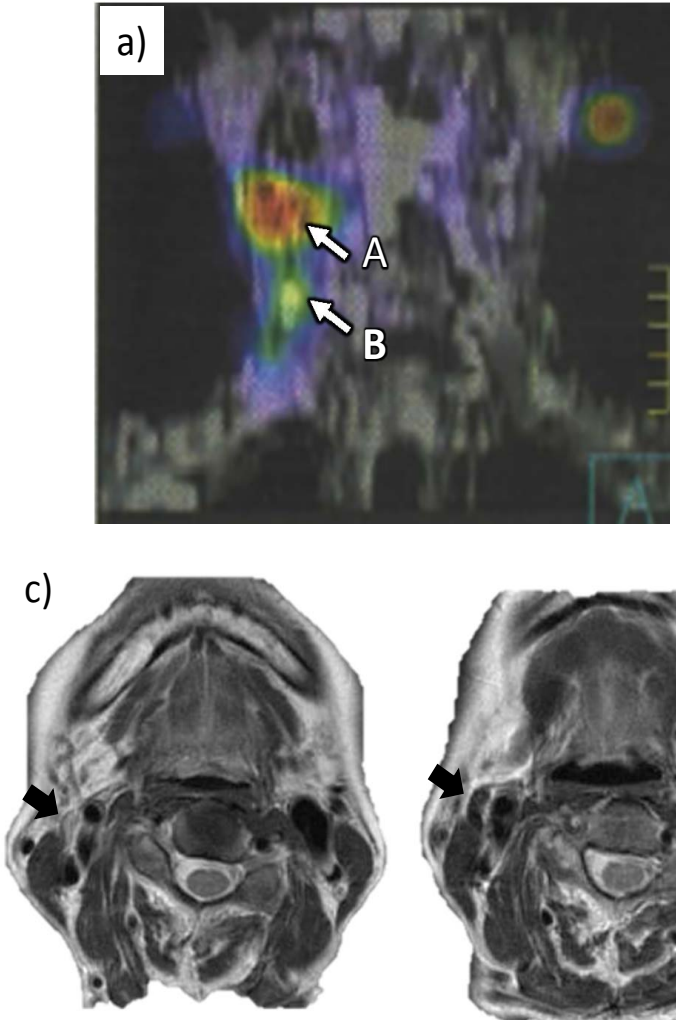

Before injection of SPIO

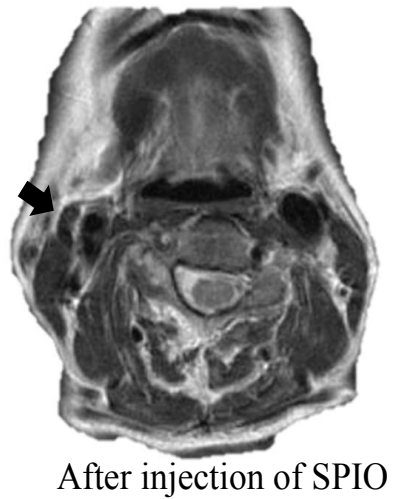

b)

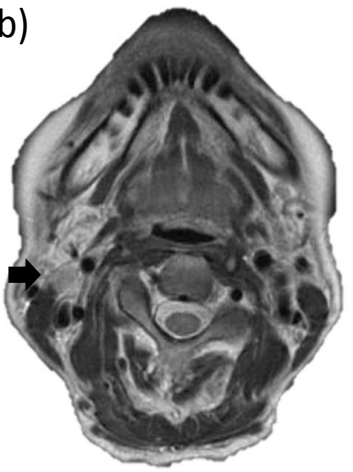

Before injection of SPIO

d)

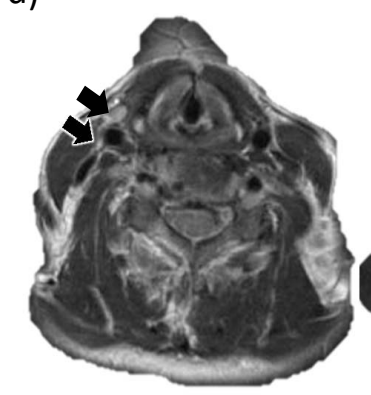

Before injection of SPIO

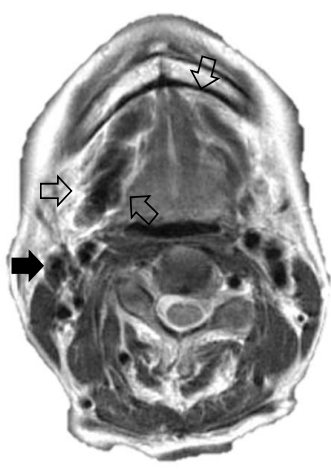

After injection of SPIO

Fig. (2). Case1; a) The locations of SLNs were identified by SPECT. b and c) The signal attenuation was shown by MRI (T2W, closed arrows). SPIO around the primary lesion was surrounded by open arrows. Those lymph nodes anatomically coincided with the hot spot A in photo a. d) Other lymph nodes with signal attenuation were detected by MRI (T2W, closed arrows). They were anatomically coincided with the lower part of hot spot B in photo a.

CT lymphangiography is one of the useful identification methods for the sentinel lymph nodes and the descriptions such as MPR and 3D are easy to understand anatomically. Saito et al. reported the lateral lingual lymph node that was identified as a sentinel node demonstrated on CT lymphography $[10,12]$. However, the timing is the key to image SLNs appropriately, as the CT contrast agent runs through the tissues in several minutes. But other modalities such as ICG method are necessary for identifying the location of SLNs during surgical operation as well.

Even with clear laterality of head and neck primary lesions, SNLs might be in the contralateral neck. Thinking of possibility that unexpected SLNs exist as previously mentioned, an isotope method has the advantage of the whole neck scan. However, there should be various methods for identifying the location of SLNs as not all of institutions can perform SPECT.

SLNs detection using SPIO is not one of the most accepted methods so far. SPIO has some problems to solve such as high price, side effects and so on. Mack et al. reported evaluation of neck metastasis by MRI with ultrasmall SPIO (USPIO) injected intravenously [13]. According to the report, MR diagnosis was correct in 26 of 27 patients who underwent surgery. Harisinghani et al. reported the evaluation of nodal metastasis in prostatic cancer patients by MRI with intravenous USPIO injection as well [14]. And they mentioned that MRI with USPIO correctly identified all patients with nodal metastases, and a node-by-node analysis had a significantly higher sensitivity than conventional MRI. Only a few were reported about submucosal injection of SPIO and its distribution $[11,15]$.

This study showed that the lymph nodes detected by MRI anatomically coincided to SLNs detected by SPECT. The uptake of SPIO in the sinus of the SLNs was pathologically proved by Fe stain. Thus, MRI using SPIO submucosal injection could detect the identical lymph nodes that were detected by SPECT.

Performing MRI before and after submucosal injection of SPIO is a complicated process. SPIO is high-priced as well. It is also expected that a precise magnetometer becomes affordable and common. Interstitial MR lymphography using SPIO is thought to be feasible for detecting SLNs in head and neck cancer patients even though there are some problems to solve for now.

\section{CONCLUSION}

We evaluated images of interstitial MR lymphography with SPIO in oral cancer patients who also underwent lymphoscintigraphy. The lymph nodes that accumulated SPIO were identical to those detected by SPECT using Tc$99 \mathrm{~m}$ phytate. SPIO can be a novel tracer for performing SLNNS and SLNB in patients with oral cancer even though it is necessary to overcome several problems. 

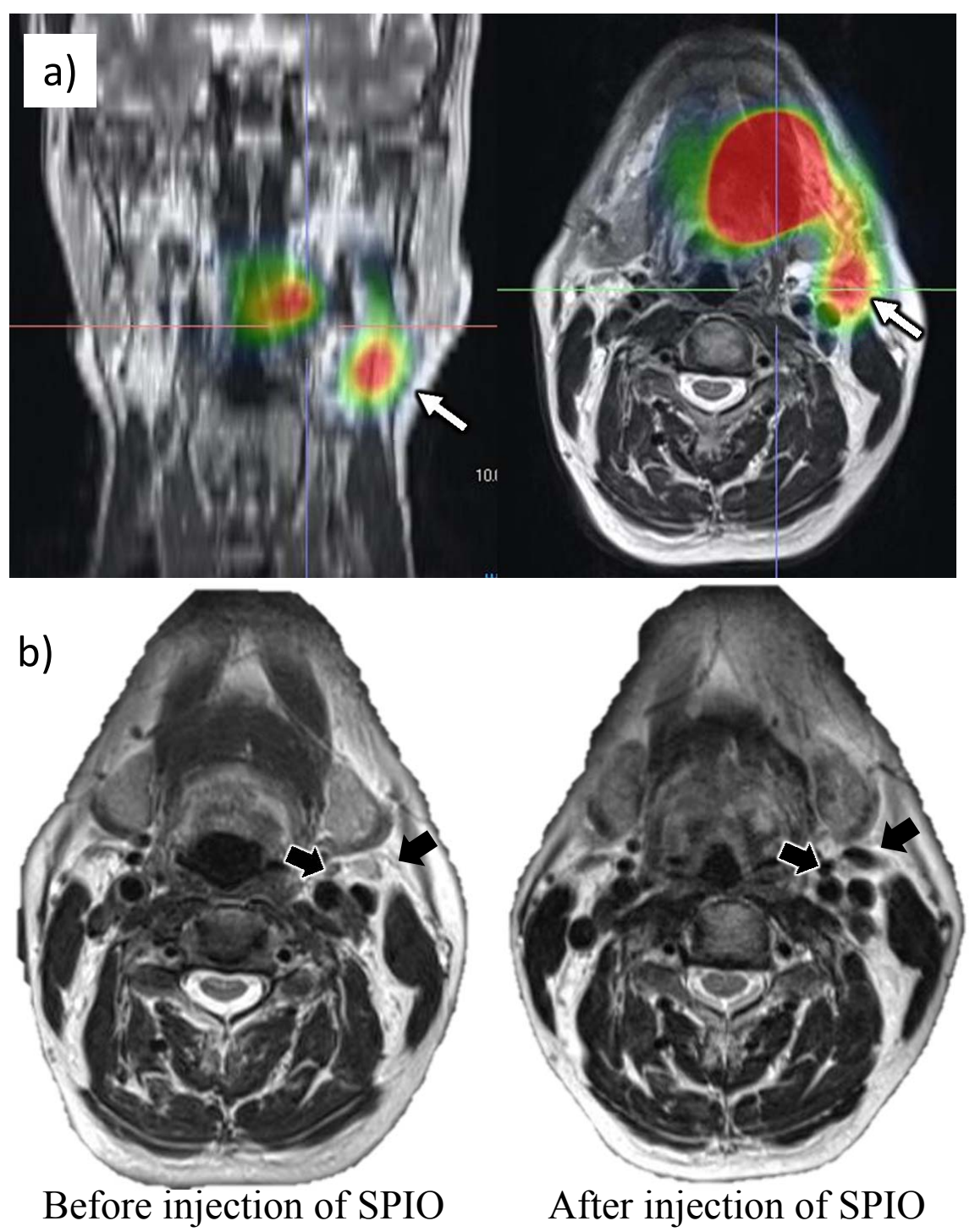

Fig. (3). Case2; a) The location of SLNs was identified in the left upper neck by SPECT (open arrows). b) The signal attenuation was shown by MRI (T2W, closed arrows). The area with decreased signal was anatomically coincided with the hot spot in photo a.

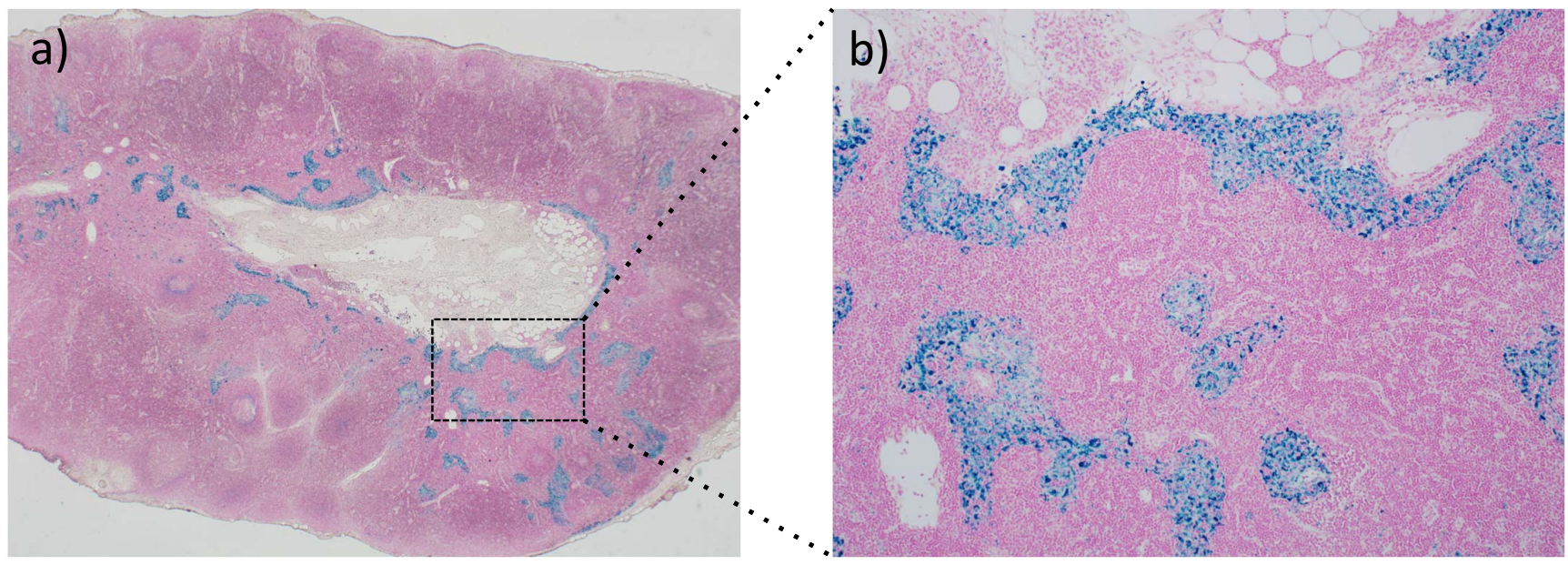

Fig. (4). A section of the lymph node detected as a SLN by a gamma-probe in Case 1. Berlin blue stain dyes iron blue in the tissue. The photos (a: x20; b: x100) show that the blue granules in sinuses are SPIO particles phagocytosed in the node. 


\section{CONFLICT OF INTEREST STATEMENT}

The authors confirm that this article content has no conflict of interest.

\section{ACKNOWLEDGEMENTS}

This study was partially supported by the grant of Osaka Foundation for the Prevention of Cancer and Cardiovascular Diseases as well as the Ministry of Health, Labour and Welfare, Grant-in-Aid for Scientific Research, 2009-2011 (201119016).

\section{REFERENCES}

[1] McMasters KM, Reintgen DS, Ross MI, et al. Sentinel lymph node biopsy for melanoma: controversy despite widespread agreement. J Clin Oncol 2001; 19: 2851-5.

[2] Krag DN, Weaver DL, Alex JC, et al. Surgical resection and radiolocalization of the sentinel lymph node in breast cancer using a gamma probe. Surg Oncol 1993; 2: 335-9; discussion 340.

[3] Giuliano AE, Kirgan DM, Guenther JM, et al. Lymphatic mapping and sentinel lymphadenectomy for breast cancer. Ann Surg 1994; 220: 391-8; discussion 398-401.

[4] Civantos FJ, Zitsch RP, Schuller DE, et al. Sentinel lymph node biopsy accurately stages the regional lymph nodes for T1-T2 oral squamous cell carcinomas: results of a prospective multiinstitutional trial. J Clin Oncol 2010; 28: 1395-400.

[5] Yoshimoto S, Hasegawa Y, Matsuzuka T, et al. Sentinel node biopsy for oral and laryngopharyngeal squamous cell carcinoma: a retrospective study of 177 patients in Japan. Auris Nasus Larynx 2012; 39: 65-70.

[6] Ogasawara Y, Ikeda H, Takahashi M, et al. Evaluation of breast lymphatic pathways with indocyanine green fluorescence imaging in patients with breast cancer. World J Surg 2008; 32: 1924-9.
[7] Yamauchi K, Nagafuji H, Nakamura T, et al. Feasibility of ICG fluorescence-guided sentinel node biopsy in animal models using the HyperEye Medical System. Ann Surg Oncol 2011; 18: 2042-7.

[8] Tagaya N, Nakagawa A, Abe A, et al. Non-invasive identification of sentinel lymph node using indocyanine green fluorescence imaging in patients with breast cancer. Open Surg Oncol J 2010; 2: 71-4.

[9] Minamiya $\mathrm{Y}$, Ito M, Katayose $\mathrm{Y}$, et al. Intraoperative sentinel lymph node mapping using a new sterilizable magnetometer in patients with nonsmall cell lung cancer. Ann Thorac Surg 2006; 81: 327-30.

[10] Shiozawa M, Lefor AT, Hozumi Y, et al. Sentinel lymph node biopsy in patients with breast cancer using superparamagnetic iron oxide and a magnetometer. Breast Cancer 2013; 20: 223-9.

[11] Mizokami D, Kosuda S, Tomifuji M, et al. Superparamagnetic iron oxide-enhanced interstitial magnetic resonance lymphography to detect a sentinel lymph node in tongue cancer patients. Acta Otolaryngol 2013; 133: 418-23.

[12] Saito M, Nishiyama H, Oda $\mathrm{Y}$, et al. The lingual lymph node identified as a sentinel node on CT lymphography in a patient with cN0 squamous cell carcinoma of the tongue. Dentomaxillofac Radiol 2012; 41: 254-8.

[13] Mack MG, Balzer JO, Straub R, et al. Superparamagnetic iron oxide-enhanced MR imaging of head and neck lymph nodes. Radiology 2002; 222: 239-44.

[14] Harisinghani MG, Barentsz J, Hahn PF, et al. Noninvasive detection of clinically occult lymph-node metastases in prostate cancer. N Engl J Med 2003; 348: 2491-9.

[15] Ishiyama K, Motoyama S, Tomura N, et al. Visualization of lymphatic basin from the tumor using magnetic resonance lymphography with superparamagnetic iron oxide in patients with thoracic esophageal cancer. J Comput Assist Tomogr 2006; 30: $270-5$. 